Scientific Visualization, 2021, volume 13, number 2, pages 149 - 156, DOI: 10.26583/sv.13.2.10

\title{
Expansion of the Functions of the Multi-View Stereomaker Software for Automatic Construction of Complex Stereo Images
}

\author{
S.V. Andreev ${ }^{1, A}$, N.A. Bondareva ${ }^{2, A}$, A.E. Bondarev3,A \\ Keldysh Institute of Applied Mathematics RAS \\ 1 ORCID: 00oo-0oo1-8029-1124, esa@keldysh.ru \\ 2 ORCID: 0000-0002-7586-903X, niki.99@mail.ru \\ 3 ORCID: 0000-0003-3681-5212, bond@keldysh.ru
}

\begin{abstract}
This paper presents the second version of the Multi-view StereoMaker 2.0 software package, which allows one to automatically generate complex stereo images according to userspecified parameters. Complex stereo images include both the main object and accompanying inscriptions, formulas and designations. The software package is designed to use the multiview technology on an autostereoscopic monitor and works with arbitrary $3 \mathrm{D}$ objects, presenting the results of solving mathematical modeling problems in the form of a stereo image.

An autostereoscopic monitor allows one to view stereo images without glasses, while providing quality that rivals classic $3 \mathrm{D}$ projection stereo systems.

The description of the main changes and new features of the second version of the developed system is given. The results of the operation of the Multi-view StereoMaker software complex on real problems are presented.
\end{abstract}

Keywords: stereo images, autostereoscopic monitor, multi-view, software.

\section{Introduction}

Nowadays, such an area of research as the use of stereo-animation technologies in various areas of life, in particular, the possibility of using them in scientific developments, is acquiring intensive development. The use of modern stereo devices can significantly improve the visual component when using them to visualize the results of numerical experiments.

A three-dimensional representation of the process evolution over time helps to verify the computational model and the algorithms used. It also provides the observer with a full understanding of the phenomenon being modeled and popularizes the research itself and its results for society, including for decision-makers.

In contrast to a two-dimensional flat image, three-dimensional stereo allows one to more effectively explore and model objects with a developed spatial structure or interpret photographs taken during an experiment from different angles at given points in time and combined into animation.

With the emergence of scientific interest in the field of stereotechnology, more and more works began to appear on various aspects of this vast area of research. Specific problems arising when using a computer system to generate and visualize a composite multiscreen stereo frame, and methods for solving such problems are described in sufficient detail in [1-3]. Initially, most of the research in the field of stereo images related to the problems of constructing virtual reality, simulators and simulators, as well as training systems. However, later, the issue of creating presentation complexes became very relevant, which, among other things, made it possible to demonstrate the results of scientific research in stereoscopic mode. These works include papers [3-10]. The paper [3] presents the results of displaying the Supernova explosion in stereo mode. The paper [7] is devoted to the creation of a computational technology for modeling the operation of a three-dimensional unit of the blades of a power plant 
in a viscous compressible heat-conducting gas flow. Currently, the tasks of constructing stereo images are considered in various fields of research [3-9]. The problems of visual discomfort in virtual and mixed reality systems are described in detail in [17].

Stereo imaging is possible using a fairly wide range of different technologies: from classical stereoscopy to depth maps. The choice of technology depends on the type of stereo devices available, the intended field of application and the actual objectives of the stereo imaging.

It should be noted that when constructing a stereo image of scientific calculations results at a certain moment, a rather relevant structural subproblem arises. The standard presentation of a scientific report, as a rule, includes not only the visualization of complex geometry or fields of physical quantities, but also explanations, usually represented by text and formulas. If for geometries and physical quantities the issues of constructing stereo presentations have been sufficiently developed, then the problems of combining them with stereo images of texts and formulas with sufficient expressiveness and the necessary stereo effect are still open.

\section{Background}

This work continues the cycle of research works [1-3,7,11-14,15,18,19] carried out at Keldysh Institute of Applied Mathematics of the Russian Academy of Sciences based on the existing stereo devices.

The main type of device for which the software system for creating stereo images described in this work is intended is Dimenco DM654MAS autostereoscopic monitor. Autostereoscopic monitors provide stereo images without the need to track the position of the observer. Typically, such monitors allow to view stereo images, providing several fixed segments in the space for observation. The viewer can move between segments, getting the opportunity to view the demonstrated object in $3 \mathrm{D}$ from different angles of view.

One of the important tasks that become relevant with the spread of such stereo devices is the combination of images and text information in one frame. Many objects, especially those that represent the results of scientific research, require accompanying information located directly in the same frame as the image. In many cases, the object and the signature, in principle, cannot be separated into different frames, since they constitute a single logical display of information. It is this task that the Multi-view StereoMaker software complex, described in detail in [18], is designed to help solve.

In the process of research, analyzed in papers [12,13], where a step-by-step process of developing such a technology based on multi-view presentation was considered, the following technology for constructing a stereo frame was worked out, which is performed manually by the user:

1) Rotation of the object (the result of visualization of scientific calculations) along the $Z$ axis by a given degree and the subsequent saving of the frame with each rotation;

2) Creation of 9 frames for each rotation: 1st frame - the leftmost position, 9 - the rightmost;

3) Adding the necessary text (with volume and clarity) for each frame;

4) Consecutive rotation of the text by the required fraction of a degree in each frame;

5) Stitching all frames into a matrix of images in a certain sequence;

The sequential execution of each of the actions of this seemingly simple algorithm, however, when executed manually, requires great care and takes an inordinately long time to perform essentially the same operations. Such an algorithm requires automation to speed up the process at times.

For these purposes, the Multi-view StereoMaker software package was developed. It allows you to construct the necessary nine views for each of the objects in a complex frame, and then combine them without violating the integrity of the final stereo image, greatly facilitating the creation of full-fledged stereo images. 
The first version of the program was registered in 2020 [19]. At the moment, the research continues to improve the capabilities of the program. This article describes the second version of Multi-view StereoMaker and its new capabilities.

\section{Key features and new options of the Multi-view StereoMaker 2.o software package}

Multi-view StereoMaker allows one to create a set of images - representations of the rotation of three-dimensional objects on a given angular stereobase - and then obtain a stereo image combined from these images.

The program is designed to work with arbitrary $3 \mathrm{D}$ objects with specified parameters. Such objects can be the results of scientific calculations and accompanying information to them, such as accompanying inscriptions, formulas, additional images. Multi-view StereoMaker implements interaction with the Tecplot software package, as well as with the Blender software package for generating arbitrary $3 \mathrm{D}$ images.

The main functions performed by the first version of the program: selection of the stereo image generation algorithm for each object, the ability to preview the generated images according to the specified parameters for each object, setting the parameters for superimposing images on top of each other at the coordinates specified by the user, as well as choosing the way to save the final result.

Among the algorithms for generating stereo images, there is a category of "custom images", where the user, respectively, can specify the directory in which his images are located. When choosing the "Tecplot" category, the user must specify a .plt file with the required model and set the desired parameters. The last category - the Blender algorithm - assumes the generation of a $3 \mathrm{D}$ object from the original image.

In the second version of the program, the interface of which is shown in Figure 1, the choice of possible algorithms for generating a stereo image is expanded, the ability to interactively enter text from the screen with its subsequent transformation into a $3 \mathrm{~d}$ object is added. This allows the user to work faster and more efficiently with the program and create the desired stereo images with accompanying information.

Also, an algorithm for automatic generation of the final result into an intermediate format was implemented, which is necessary for further reformatting the result into a specialized .d9v format, with which the autostereoscopic monitor interacts. This will speed up the user work with stereo installation, eliminating several iterations from the chain of format conversions.

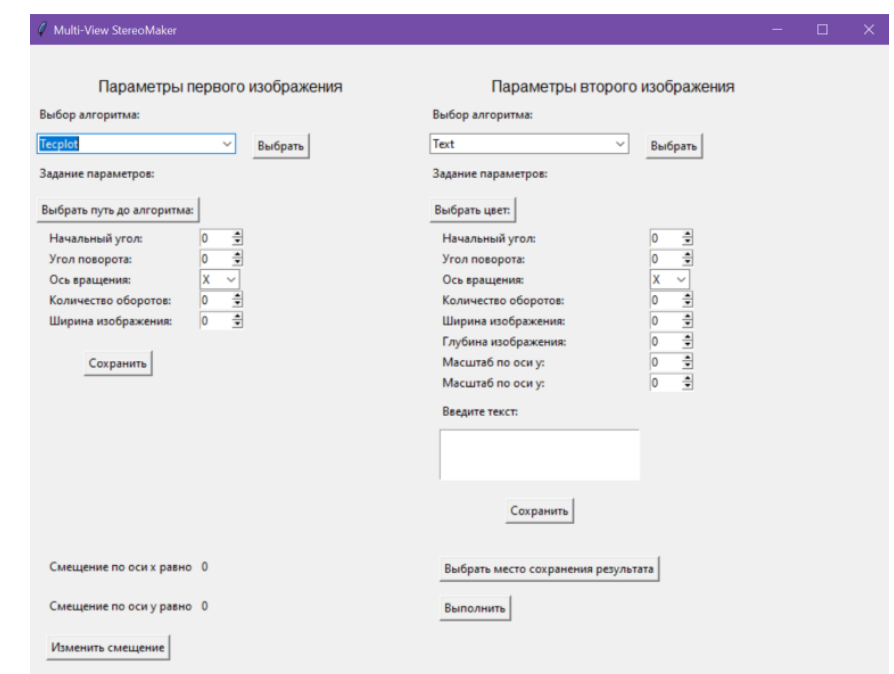

Fig.1. Multi-view StereoMaker 2.o software package interface. 
Multi-view StereoMaker 2.0 consists of the following components, shown in the diagram (Fig. 2). The user must enter the parameters using the provided graphical interface (GUI). The graphical interface allows one to access the generated images preview subsystem for each object. The parameters entered by the user are passed to the preliminary data subsystem, which creates a directory system for storing the results of the algorithms. Then the parameters are passed to the algorithm generator. Then the finished result is processed and properly formatted.

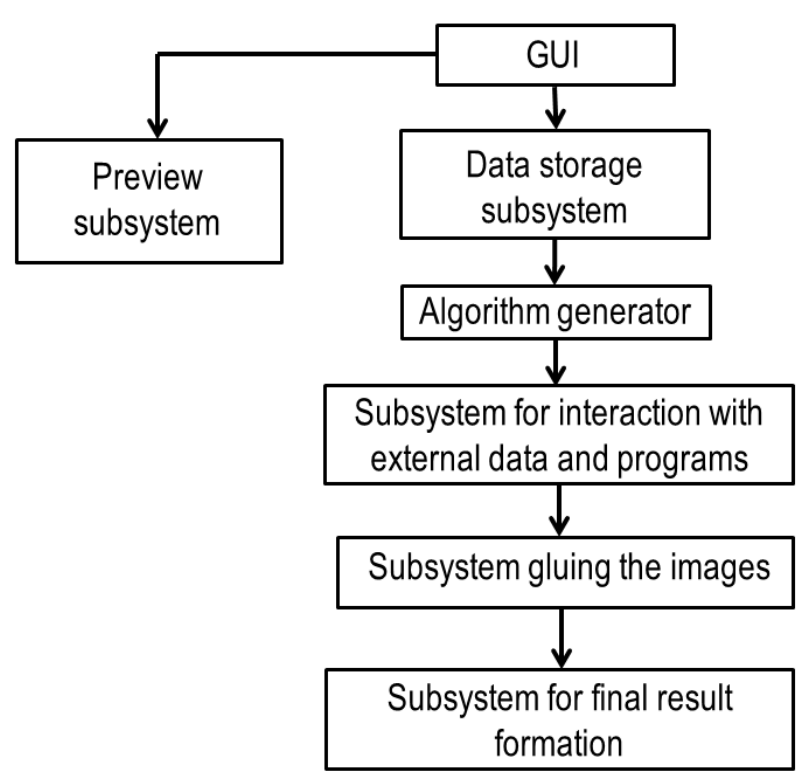

Fig.2. Organization scheme of the Multi-view StereoMaker software package.

The algorithm generator generates the appropriate algorithms for the operation of external programs (Tecplot, Blender), after which the obtained algorithms and parameters are transferred to the subsystem for interaction with external programs and data. Then the image fusion subsystem is called, which performs multiple superposition of a number of images formed for two objects. The result of the program's work is a directory containing algorithms for external programs generated for a specific case, directories with generated images for objects with user-specified parameters, and a directory with the final result.

The system was tested on the real results of a generalized computational experiment on the comparative assessment of the accuracy of three solvers (Fig. 3-5) of the open software package OpenFOAM on the problem of supersonic flow around a cone at an angle of attack [20].

The generalized computational experiment allows carrying out parametric calculations based on the synthesis of algorithms for solving problems of mathematical modeling, parallel technologies and visual analytics. The main methods and approaches for constructing a generalized computational experiment are described in detail in [21, 22]. As a rule, the results of constructing such an experiment are multidimensional data volumes. The ability to construct stereo images of the results for target functionals is very important. 

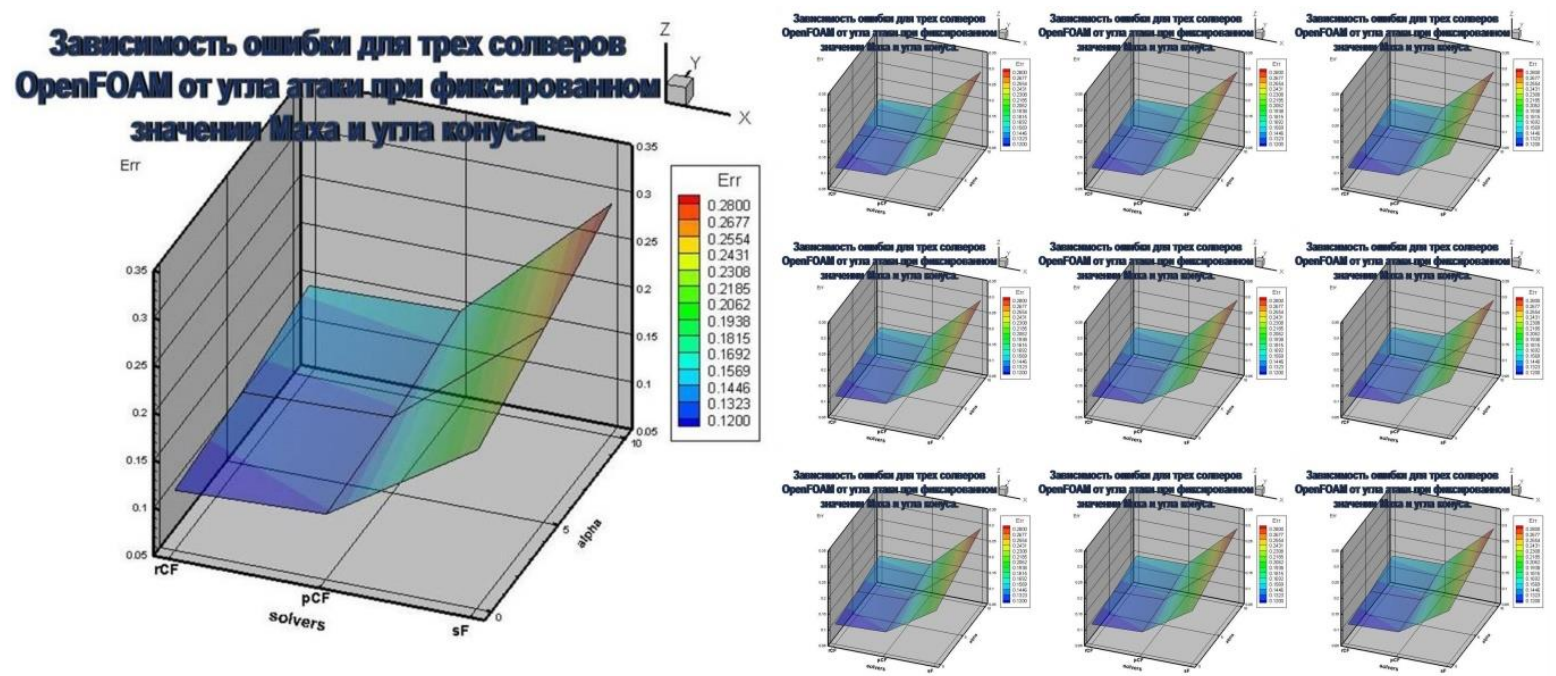

Fig.3. Dependence of the error for three OpenFOAM solvers on the angle of attack at a fixed Mach number and cone angle.
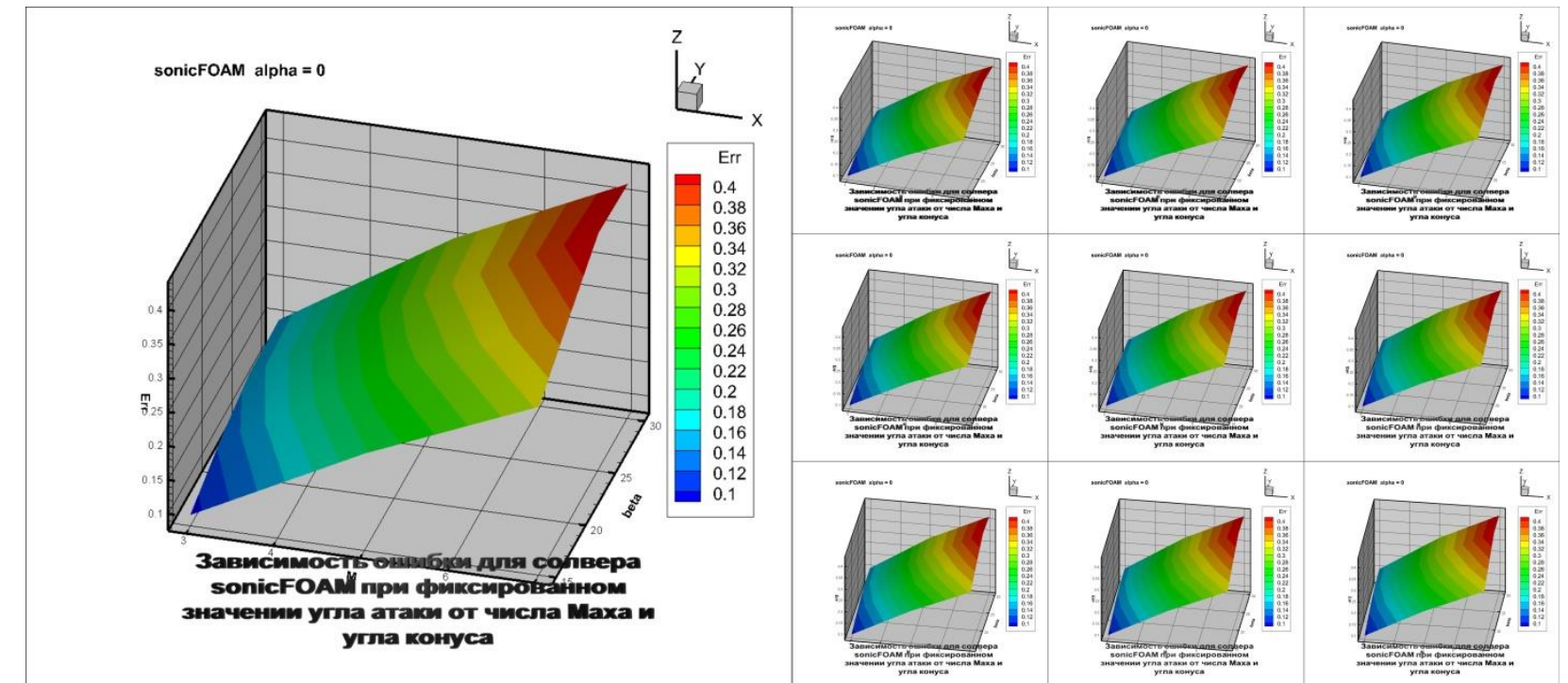

Fig.4. Dependence of the error for the sonicFOAM solver at a fixed value of the angle of attack on the Mach number and the angle of the cone
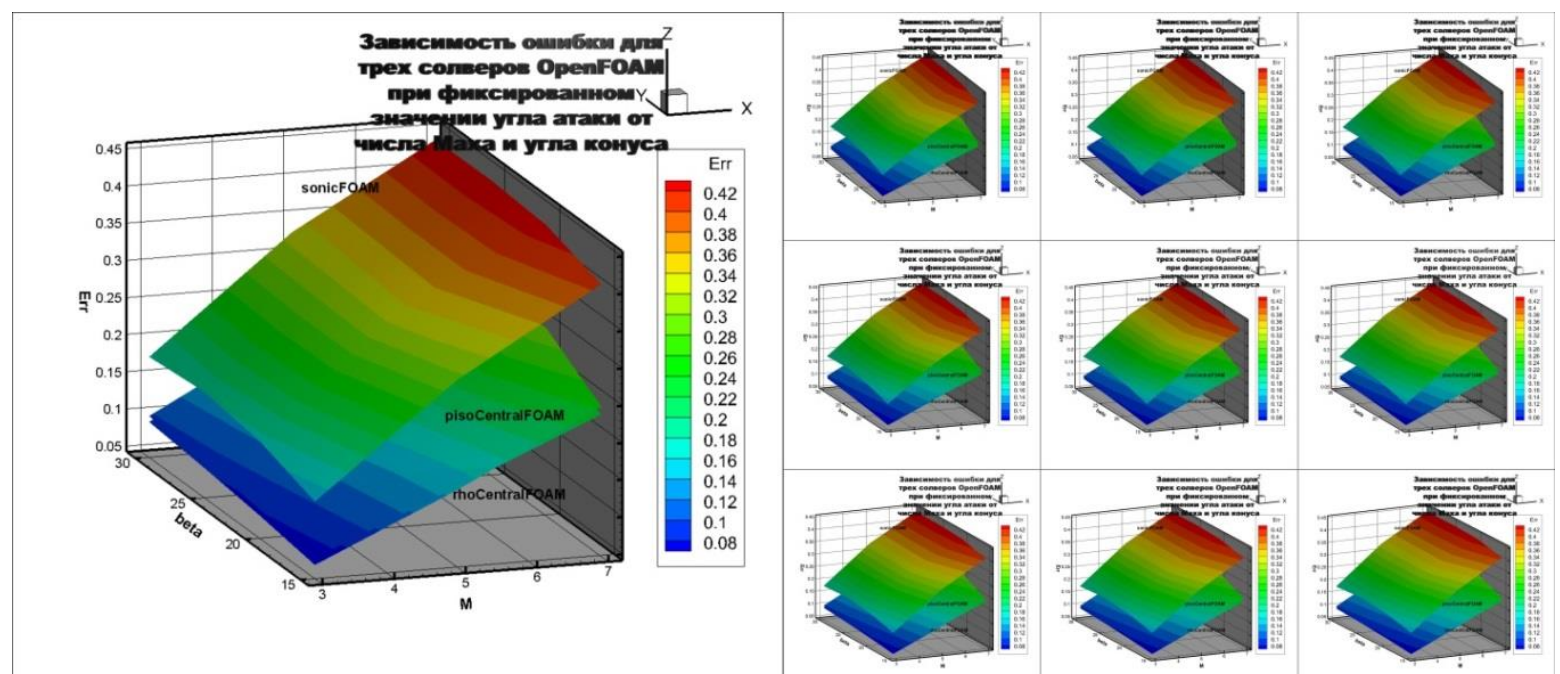

Fig.5. Dependence of the error for three OpenFOAM solvers at a fixed value of the angle of attack on the Mach number and the angle of the cone. 
The Multi-view StereoMaker project is incomplete. It is planned to further develop and improve the program for combining several objects and interactively placing them in a stereo frame with the simultaneous generation of nine views for each. Also, additional options will be developed for the accounting of statistical data.

\section{Conclusion}

The article presents the results of constructing a stereo image for an autostereoscopic monitor by the method of multi-view representation using the second version of the Multiview StereoMaker 2.0 software package.

Additions have been made to effectively improve the generation of stereo images with textual accompaniment for creating stereo presentations. There was also a demonstration of the program's work on specific real problems.

In the future, it is planned to further develop custom functions, as well as to solve the problem of interactively placing objects in one stereo frame with the simultaneous generation of nine views for each.

\section{References}

[1] Synthesis of photorealistic three-dimensional images in modern presentation systems/ Andreev S.V.[et al] // Software\&Systems 2007. № 3. p. 37-40. [in Russian].

[2] Generation of Stereo-Presentations in Photorealistic Rendering and Scientific Visualization /Andreev S.V.[et al] // Keldysh Institute preprints, 2010. № 61. 16 p. http://library.keldysh.ru/preprint.asp?id=2010-61[in Russian].

[3] Andreev S., Filina A. Using stereo presentations for visualization of scientific calculations results / Scientific Visualization. 2012. v.4. № 1. p.12-21

[4] Hardware-software complex of 3D presentations based on a virtual studio and virtual environment / Vandanov V.G. [et al] // Proceedings of the 1-st international conference «3D visualization of scientific, technical and social reality. Cluster technologies of model-ing» Izhevsk. 2009. P.73-77. [in Russian].

[5] Mezhenin A.V., Tozik V.G. 3D Visualization using the stereo image effect // Proceedings of the 2-nd international conference $" 3 \mathrm{D}$ visualization of scientific, technical and social reality. Cluster technologies of modeling» Izhevsk. 2010. [in Russian].

[6] Mikhaylyuk M.V., Huraskin I.A. Synthesis of stereo images for virtual reality systems using an optical tracking system / Software\&Systems 2006. № 3. p. 10-14. [in Russian].

[7] Andreev S.V. [et al] Modelling and visualisation of blade assembly with complicated shape for power turbine / Scientific Visualization. 2015. v.7. № 4. p.1-12

[8] Torgashev M.A., P.Y. Timokhin. The technology of stereo video files' synthesis for the system of 3D real-time visualization. Software Products and Systems, 2012, № 3, pp. 74-80. [In Russian]

[9] Mikhaylyuk M.V., Maltsev A.V. Timokhin P.Yu. The methods of 3D stereo visualizationof data obtainedin simulation of unstable oil displacement from porous media / Proceedings of Scientific Research Institute for System Analysis RAS, 2018, v.8, N 2, p. 125-129. [in Russian].

[10]Visibility Experiment and Evaluation of 3D Character Representation on Mobile Displays / Hiromu Ishio [et al.] // C. Stephanidis (Ed.): Posters, Part II, HCII 2011, CCIS 174, pp. 46-51, 2011.

[11]Andreev S.V., Bondareva N.A. Constructing a representation of textual information in stereo presentations // Proceedings of the 28-th International Conference of Computer Graphics and Vision GraphiCon-2018, Tomsk, Russia, 23-28 September 2018, p. 86-89. [in Russian].

[12]S.V.Andreev, A.E.Bondarev, V.A.Galaktionov, N.A.Bondareva (2018) The problems of stereo animations construction on modern stereo devices. Scientific Visualization 10.4: 40 52, DOI: $10.26583 /$ sv.10.4.04 
[13]S.V. Andreev, N.A. Bondareva, E.Yu. Denisov. Stereo Presentations Problems of Textual information on an Autostereoscopic Monitor (2019). Scientific Visualization 11.4: 90 101, DOI: 10.26583/sv.11.4.08

[14]S.V. Andreev, A.E. Bondarev, N.A. Bondareva. Stereo images of error surfaces in problems of numerical methods verification (2020). Научная визуализация 12.2: 151 - 157, DOI: $10.26583 / \mathrm{sv} .12 .2 .12$

[15] Stereo animation "Modeling and visualization of the work of a power plant having complex shape in the stereo animation mode". https://yadi.sk/i/3CcYhlMB3TCH4r (Accessed 01.11.2020).

[16]S.V. Andreev, A.E. Bondarev, N.A. Bondareva. Stereoscopic construction of textual information in presentations of research results on an autostereoscopic monitor (2020). Scientific Visualization 12.1: 132 - 139, DOI: 10.26583/sv.12.1.12

[17]A.D. Zhdanov, D.D. Zhdanov, N.N. Bogdanov, I.S. Potemin,V.A. Galaktionov, and M.I. Sorokin. Discomfort of Visual Perception in Virtual and Mixed Reality Systems // Programming and Computer Software, 2019, Vol. 45, No. 4, pp. 147-155. DOI: 10.1134/So36176881904011X

[18] S.V. Andreev, A.E. Bondarev, N.A. Bondareva, K.A. Donskoy, V.A. Galaktionov. Automated construction of a stereo image for results of mathematical modeling using the Multiview StereoMaker software system (2020). Scientific Visualization 12.5: 165 - 173, DOI: 10.26583/sv.12.5.12

[19] Certificate of state registration of a computer program No. 2020664486. The program for automatic construction of stereopresentation for an autostereoscopic monitor using the Multi-view method "Multi-View Stereomaker" / Bondarev A.E., Galaktionov V.A., Andreev S.V., Bondareva N.A. ., Donskoy K.A. ; applicant and copyright holder Keldysh Institute of RAS (RU). - 2020663564 app. 11/05/2020; publ. 13.11.20, Register of computer programs.

[20] Aleksey Alekseev, Alexander Bondarev, Vladimir Galaktionov, Artem Kuvshinnikov, Lev Shapiro. On Applying of Generalized Computational Experiment to Numerical Methods Verification // CEUR Workshop Proceedings, V. 2744, 2020, p. paper19-1 - paper19-12, DOI: 10.51130/graphicon-2020-2-3-19

[21]Bondarev A.E. On the Construction of the Generalized Numerical Experiment in Fluid Dynamics // Mathematica Montisnigri, Vol. XLII, 2018, p. 52-64

[22]A.E. Bondarev. On visualization problems in a generalized computational experiment (2019). Scientific Visualization 11.2: 156 - 162, DOI: 10.26583/sv.11.2.12 